\title{
Laser-induced preferential dehydrogenation of graphane
}

\author{
Hong Zhang, ${ }^{1}$ Yoshiyuki Miyamoto, ${ }^{2, *}$ and Angel Rubio ${ }^{3}$ \\ ${ }^{1}$ College of Physical Science and Technology, Sichuan University, Chengdu 610065, China \\ ${ }^{2}$ Graphene Division, Technology Research Association for Single Wall Carbon Nanotubes (TASC), Nanosystem Research Institute, \\ National Institute of Advanced Industrial Science and Technology (AIST), Central 2, 1-1-1 Umezono, Tsukuba 305-8568, Japan \\ ${ }^{3}$ Nano-Bio Spectroscopy group and ETSF Scientific Development Centre, Centro de Física de Materiales CSIC-UPVIEHU-MPC and DIPC, \\ Universidad del País Vasco UPVIEHU, Avenida de Tolosa 72, E-20018 San Sebastian, Spain
}

(Received 19 February 2012; published 18 May 2012)

\begin{abstract}
We have used first-principles simulations based on time-dependent density functional theory to show that short laser pulses can trigger preferential hydrogen desorption from the upper or lower side of suspended graphane (H-terminated graphene). This control is achieved by using intense ultrashort $p$-polarized laser pulses $(\sim 2 \mathrm{fs})$ with an asymmetric time envelope. The dynamical Stark effect induced by the pulse creates an asymmetric charge distribution and force field on the $\mathrm{H}$ ions, even at low laser fluence. At finite temperatures the carbon-hydrogen stretching softens, favoring $\mathrm{H}$ desorption from one side. This transient geometry can be modified by halogen functionalization, which results in a two-dimensional dipolar structure.
\end{abstract}

DOI: 10.1103/PhysRevB.85.201409

PACS number(s): 81.05.Zx, 68.43.Tj, 81.05.U-, 82.53.St

Single-layer graphene is fabricated by mechanically peeling off a layer of carbon from pyrolytic graphite. ${ }^{1}$ Graphene's unique electronic, chemical, and mechanical properties and its potential applications have been extensively studied. Chemically modified graphene has been investigated, ${ }^{2}$ and hydrogen $(\mathrm{H})$-terminated graphene, known as graphane, has also been found to be theoretically stable. ${ }^{3}$ Further theoretical studies have examined its structural, magnetic, and optical properties, ${ }^{4-9}$ and its experimental synthesis has also been investigated. ${ }^{10,11}$ Graphene that is $\mathrm{H}$ terminated on only one side has also been proposed for use in spin-polarized devices with the structure known as graphone, ${ }^{12}$ or as a gapped semiconductor material with full $\mathrm{H}$ coverage. ${ }^{13}$

In this Rapid Communication we propose a way of $\mathrm{H}$ desorption, which is mainly from one side of a suspended graphane sheet, with the use of an asymmetric pulse of the femtosecond laser by performing first-principles simulations. Further chemical modification of this side with other reactive species can reach the heterogeneously terminated graphene, which was recently studied. ${ }^{14}$ We emphasize that this asymmetrically biased desorption is thermodynamically difficult and thus has not been considered in former theoretical studies for stability, ${ }^{15,16}$ because the carbon-hydrogen $(\mathrm{C}-\mathrm{H})$ bond is the same on both sides. The femtosecond laser pulse has an asymmetric electric field ( $E$-field) time envelope in order to induce dehydrogenation on one side of the graphane in our first-principles molecular dynamics calculations, based on the Ehrenfest time-dependent density functional theory (E-TDDFT) framework. ${ }^{17,18}$ In this work, the $z$ axis is set normal to the graphane sheet with the sheet at $z=0$, and $z>0$ and $z<0$ are taken as the upper and lower regions, respectively (Fig. 1). An $E$-field polarized normal to the graphane sheet with the envelope shown in Fig. 2 results in a positive (upward) force on the ions and a negative force on the electrons. The $\mathrm{H}$ atoms in the upper region are desorbed from the sheet by this pulse, whereas the $\mathrm{H}$ atoms in the lower region remain bound. Thus, the upper graphene region remains chemically active, making this graphone sheet transient, which can be used for further functionalization. ${ }^{19}$ We provide a complete microscopic description of this process, which may have implications beyond the present study. The one-sided $\mathrm{H}$ desorption is caused by the asymmetric force field and charge density induced by the laser pulse, known as the dynamical Stark effect. The energy transfer, called the effective fluence, from the laser pulse to the graphane in our simulations was of the order of $10 \mathrm{~mJ} / \mathrm{cm}^{2}$. The numerical estimation of laser fluence through the simulation of a short-laser pulse has previously been investigated. ${ }^{20}$

In the present simulation, we examine the proposed most stable chair conformation ${ }^{3}$ of graphane. A laser pulse with a main driving wavelength of $800 \mathrm{~nm}$ and a full width at half maximum of $2 \mathrm{fs}$ is used (Fig. 2). The pulse-induced electron-ion dynamics is described by coupling TDDFT for the electron time evolution with classical molecular dynamics (MD) for the ions (Ehrenfest dynamics ${ }^{21}$ ), ${ }^{17}$ as implemented in the first-principles simulation tool for electron-ion dynamics (FPSEID) code. The Suzuki-Trotter split operator technique ${ }^{22}$ was used for the real-time propagation of the Kohn-Sham wave functions. The optical $E$ field is described in the length gauge by a time-dependent scalar potential $V_{\text {ext }}(\mathbf{r}, t)$ in the Kohn-Sham Hamiltonian. ${ }^{23,24}$ This scheme allows the time-dependent optical field to interact with both ions and electrons throughout the simulation and the identification of the microscopic mechanisms responsible for the controlled desorption of $\mathrm{H}$ atoms. The time-dependent Kohn-Sham equations,

$$
i \hbar \frac{d \psi_{n}(\mathbf{r}, t)}{d t}=\left[H_{\mathrm{KS}}(\mathbf{r}, t)+V_{\mathrm{ext}}(\mathbf{r}, t)\right] \psi_{n}(\mathbf{r}, t),
$$

are coupled to the Newton equations for the ions. Here $H_{\mathrm{KS}}(\mathbf{r}, t)$ is the Kohn-Sham Hamiltonian, which is a functional of the time-varying charge density $\rho(\mathbf{r}, t)$, which is uniquely determined from the time- and spatial-dependent external field ${ }^{25} \psi_{n}(\mathbf{r}, t)$ are the corresponding time-dependent $n$th Kohn-Sham orbitals, from which the electron density is obtained by summing over the occupied Kohn-Sham orbitals. During the MD simulation under the external optical $E$ field, the conservation of total energy (electronic plus ionic) minus the work done by the optical $E$ field $^{26}$ is found. Periodic boundary conditions are applied, therefore, a supercell 


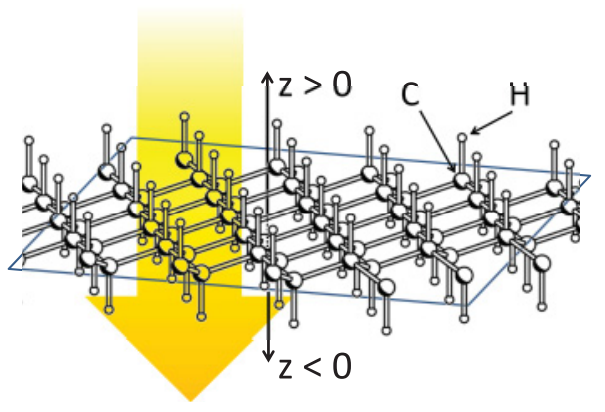

FIG. 1. (Color online) Schematic showing the asymmetric time average for the applied optical electric field ( $E$ field) of the laser pulse. The polarization of the $E$ field is normal to the graphane sheet and its positive direction corresponds to the upper graphane sheet, $z>0$. Hydrogen and carbon atoms are denoted by small open circles and shaded circles, respectively.

consisting of a $1 \times 1$ graphane unit cell plus a vacuum region of $10 \AA$ is used in order to avoid spurious interactions between cells. $^{27}$ The interaction between valence electrons and ions are described by norm-conserving pseudopotentials, ${ }^{29}$ which we have previously used for the nonequilibrium dynamics of carbon-based materials. ${ }^{30}$ Our optimized lattice constant, which was $2 \%$ larger than that of graphene, and the internal atomic coordinates of graphane are consistent with literature values. ${ }^{3}$

A laser pulse with polarization normal to the graphane sheet is applied to graphane in the optimized (zero temperature) ground-state geometry. This laser polarization corresponds to the $p$ polarization of light with its incident angle off the surface normal. The optical field is expressed by a sawtooth potential along the normal direction, which preserves the spatial periodicity of the supercell in $V_{\text {ext }}(\mathbf{r}, t)$ in Eq. (1), which contains a polarity inversion in the middle of the vacuum region.

Figure 2 shows the asymmetric forces acting on the $\mathrm{H}$ atoms throughout the pulse duration. The asymmetric force field can be explained by the laser-induced dynamical Stark effect,

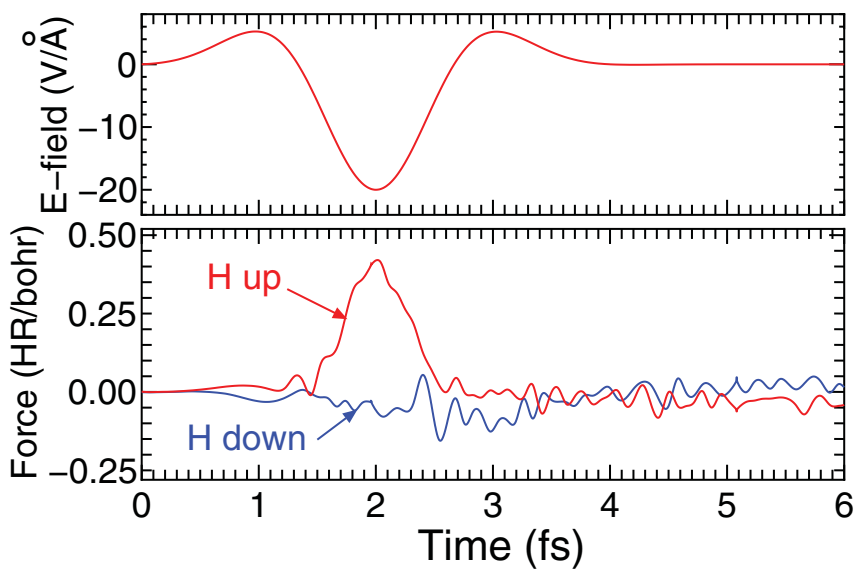

FIG. 2. (Color online) Upper: The time evolution of the applied pulse field with $E_{\max }=20 \mathrm{~V} / \AA$. The direction is defined for the force on the negatively charged particle, and the positive and negative directions correspond to $z>0$ and $z<0$ regions in Fig. 1 , respectively. Lower: The time evolution of forces on the upper $(z>0)$ and lower $(z<0)$ hydrogen atoms.
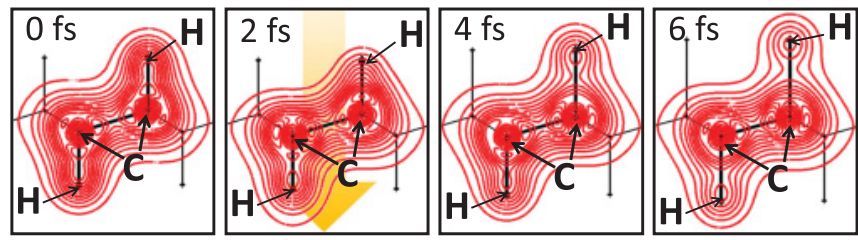

FIG. 3. (Color online) Time evolution of an electron cloud where $E_{\max }=20 \mathrm{~V} / \AA$. The charge contour lines on a plane normal to graphane layer are plotted at $t=0,2,4$, and $6 \mathrm{fs}$. The orange arrow at $2 \mathrm{fs}$ denotes the maximum intensity of the laser field pushing the electron cloud downward.

which creates an asymmetric charge distribution. Figure 3 shows snapshots of the behavior of the electron density at $t=0,2,4$, and 6 fs and $E_{\max }=20 \mathrm{~V} / \AA$. The dynamics clearly show a downward electronic motion at $t=2 \mathrm{fs}$ because of the laser $E$ field and the backward motion following the decay of the $E$ field at $t=4 \mathrm{fs}$. The dynamic asymmetric charge redistribution and the laser acting on the hydrogen atoms accelerate the upper $\mathrm{H}$ atoms more than the lower $\mathrm{H}$ atoms. Thus the asymmetry of the $E$ field causes the asymmetric dynamics of $\mathrm{H}$ atoms.

The longer time dynamics induced by the laser pulse shown in Fig. 2 are shown in Fig. 4(a) at several values of $E_{\max }=20$, 21.36, and $22.48 \mathrm{~V} / \AA$. After 30 fs at $E_{\max }=22.48 \mathrm{~V} / \AA, \mathrm{H}$ atoms in the upper region are desorbed from the sheet, whereas the $\mathrm{H}$ atoms in the lower region remain bound. Following $\mathrm{H}$ desorption, the network of $\mathrm{C}$ atoms favors a nearly planar hexagonal configuration; the $\mathrm{C}$ atoms have a nonplanar, $s p^{3}$ like configuration at the beginning of the simulation [Fig. 4(b)].

At all the values of $E_{\max }$, the $\mathrm{C}-\mathrm{H}$ bond length begins to expand or shrink to its original size at the times marked by the triangles in Fig. 4. The change in the positions of the triangles on the time axes shows a slowdown in the frequency of C-H stretching as $E_{\max }$ increases, and that the minimum
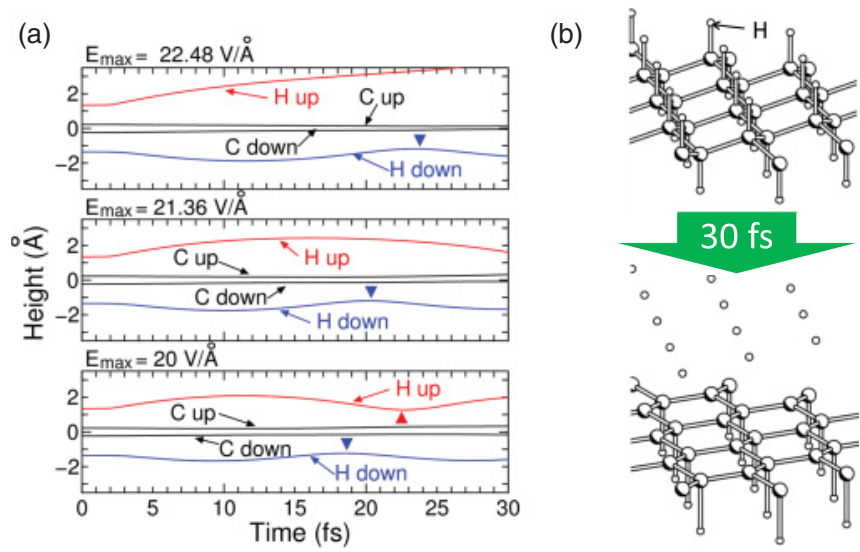

FIG. 4. (Color online) (a) The time evolution of the $\mathrm{H}$ and $\mathrm{C}$ atom height measured from the average height of the $\mathrm{C}$ atoms in the graphane sheet, for an applied laser pulse with an $E_{\max }$ of 20,21.36, and $22.48 \mathrm{~V} / \AA . \mathrm{C}_{\mathrm{up}}$ and $\mathrm{C}_{\mathrm{dwn}}$ denote the heights of $\mathrm{C}$ atoms in the upper $(z>0)$ and lower $(z<0)$ regions, whereas $H_{\text {up }}$ and $H_{d w n}$ are the corresponding heights for the $\mathrm{H}$ atoms. The blue (downward) solid triangles are $\mathrm{C}-\mathrm{H}$ stretching periods of the $\mathrm{H}$ atoms on the lower side, whereas the red (upward) triangle is the period on the upper side. (b) The desorption dynamics for $E_{\max }=22.48 \mathrm{~V} / \AA$. 
length of the upper $\mathrm{C}-\mathrm{H}$ bonds appears later than that of the lower $\mathrm{C}-\mathrm{H}$ bonds. This result suggests that the dynamical Stark effect, which causes the asymmetry, is enhanced at larger values of $E_{\max }$. When $E_{\max }$ is near the threshold of $\mathrm{H}$ desorption at $21.36 \mathrm{~V} / \AA$, the period of the upper $\mathrm{C}-\mathrm{H}$ stretching becomes significantly longer than that of lower $\mathrm{C}-\mathrm{H}$ bond stretching. When $E_{\max }=22.48 \mathrm{~V} / \AA$, the upper $\mathrm{H}$ atoms do not return to the original sheet, whereas the lower $\mathrm{H}$ atoms continue to exhibit $\mathrm{C}-\mathrm{H}$ stretching. These results suggest preferential dehydrogenation from one side of the graphane sheet at a finite temperature. However, a quantitative prediction of the dehydrogenation rate requires a quantum mechanical treatment of the proton motion, which is beyond the scope of the present study.

We next calculate the increase in the potential energy per unit cell of the graphane surface for $E_{\max }=22.48 \mathrm{~V} / \AA$. The increase in the potential energy was $27.90 \mathrm{eV}$ after the pulse had decayed, which corresponds to an effective fluence of $8.20 \mathrm{~mJ} / \mathrm{cm}^{2}$. The effective fluence depends on the periodic boundary conditions, ${ }^{20}$ although our value is well below the threshold for structural change in graphite $60 \mathrm{~mJ} / \mathrm{cm}^{2},{ }^{31}$ and for damage in graphene $89 \mathrm{~mJ} / \mathrm{cm}^{2}$ (Ref. 32) and $150 \mathrm{~mJ} / \mathrm{cm}^{2}{ }^{33}$ Recently a threshold of $14 \mathrm{~mJ} / \mathrm{cm}^{2}$ for a damage has been reported. ${ }^{34}$ These results indicate the stability of the underlying graphene sheet.

Our simulations provide a framework in which to create a transient geometry, where one side of the graphane sheet has very few or no $\mathrm{H}$ atoms. The instability of partly $\mathrm{H}$-terminated graphene was studied experimentally. ${ }^{10}$ Meanwhile, theoretical works ${ }^{15,16}$ showed the transient nature of partly $\mathrm{H}$ terminated graphene, too. Because this transient geometry remains chemically active on one side, halogen atoms could be introduced. This would create an incredibly thin dipolar film, which was theoretically studied ${ }^{14}$ and could be used in several nanoelectronics applications. Our current work validated this heterogeneously terminated structure by proposing a possible pathway of the formation. Local density approximation (LDA) calculations showed that the lattice constant of graphene that is $\mathrm{H}$ terminated on one side and fluorine $(\mathrm{F})$ terminated on the other is $3 \%$ larger than that of graphene, whereas that of $\mathrm{H}$ - and chlorine $(\mathrm{Cl})$-terminated graphene is $10 \%$ larger. Therefore, the heterogeneous termination of graphene with a mixture of
$\mathrm{H}$ and $\mathrm{F}$ atoms appears to be experimentally feasible, whereas $\mathrm{Cl}$ would be too large.

We should also address the question of what happens when the heterogeneously modified graphene sheet is supported on a pristine graphene layer. The preliminary band-structure calculation within the LDA level suggests that electron and hole transfer from the pristine graphene sheet to the heterogeneously terminated graphene takes place when the graphene sheet approaches from the $\mathrm{F}$ - or $\mathrm{H}$-terminated side. ${ }^{35}$ On the other hand, a Fermi level shift at the Dirac cone is not observed in the band structure of the pristine graphene bilayer, ${ }^{36,37}$ where the location of the Fermi level remains at the Dirac cone or the cone vanishes, depending on stacking.

In conclusion, we have demonstrated, through simulations, an appealing, efficient, and selective way to dehydrogenate a graphane sheet. Doing this is thermodynamically feasible by means of a $p$-polarized laser with an asymmetric pulse shape to irradiate graphane. A finite temperature would probably reduce the threshold for selective one-sided dehydrogenation of graphane, ${ }^{38}$ and the dehydrogenation would proceed without damage to the graphene sheet. Although the generation of a short, intense pulse $\mathrm{e}^{39,40}$ and control of the pulse shape ${ }^{41}$ may be challenging, we hope our findings will stimulate further research on controlling $\mathrm{H}$ termination on graphane in order to obtain unique chemically modified structures.

All calculations were performed by using the $\mathrm{T} 2 \mathrm{~K}$ Supercomputer System at Tsukuba University. H.Z. acknowledges financial support from the National Natural Science Foundation of China (NSFC Grant No. 11074176 and NSAF Grant No. 10976019) and support from Research Fund for the Doctoral Program of Higher Education of China (Grant No. 20100181110080). Y.M. acknowledges the fund from the Strategic Programs for Innovative Research (SPIRE), MEXT, and the Computational Materials Science Initiative (CMSI), Japan. A.R. acknowledges funding from European Research Council Advanced Grant DYNamo (ERC-2010-AdG Proposal No. 267374), Spanish projects (FIS2010-21282-C0201 and PIB2010US-00652), Grupos Consolidados UPV/EHU del Gobierno Vasco (IT-319-07), ACI-Promociona (ACI20091036), European Community project THEMA (Contract No. 228539), Ikerbasque, and SGIker ARINA (UPV/EHU).

\footnotetext{
*yoshi-miyamoto@aist.go.jp

${ }^{1}$ K. S. Novoselov, A. K. Geim, S. V. Morozov, D. Jiang, Y. Zhang, S. V. Dubonos, I. V. Grigorieva, and A. A. Fisov, Science 306, 666 (2004).

${ }^{2}$ X. Dong, Y. Shi, Y. Zhao, D. Chen, J. Ye, Y. Yao, F. Gao, Z. Ni, T. Yu, Z. Shen, Y. Huang, P. Chen, and L.-J. Li, Phys. Rev. Lett. 102, 135501 (2009).

${ }^{3}$ J. O. Sofo, A. S. Chaudhari, and G. D. Barber, Phys. Rev. B 75, 153401 (2007).

${ }^{4}$ D. W. Boukhvalov, M. I. Katsnelson, and A. I. Lichtenstein, Phys. Rev. B 77, 035427 (2008).

${ }^{5}$ O. Leenaerts, B. Partoens, and F. M. Peeters, Phys. Rev. B 80, 245422 (2009).
}

${ }^{6}$ J. Berashevich and T. Chakraborty, Phys. Rev. B 82, 134415 (2010).

${ }^{7}$ B. N. Jariwala, C. V. Ciobanu, and S. Agarwal, Phys. Rev. B 82, 085418 (2010).

${ }^{8}$ S. Lebègue, M. Klintenberg, O. Eriksson, and M. I. Katsnelson, Phys. Rev. B 79, 245117 (2009).

${ }^{9}$ P. Cudazzo, C. Attacalite, I. V. Tokatly, and A. Rubio, Phys. Rev. Lett. 104, 226804 (2010).

${ }^{10}$ D. C. Elias, R. R. Nair, T. M. G. Mohiuddin, S. V. Morozov, P. Blake, M. P. Halsall, A. C. Ferrari, D. W. Boukhvalov, M. I. Katsnelson, A. K. Geim, and K. S. Novoselov, Science 323, 610 (2009).

${ }^{11}$ J. D. Jones, K. K. Mahajan, W. H. Williams, P. A. Ecton, Y. Mo, and J. M. Perez, Carbon 48, 2335 (2010). 
${ }^{12}$ J. Zhou, Q. Wang, Q. Sun, X. S. Chen, Y. Kawazoe, and P. Jena, Nano Lett. 9, 3867 (2009).

${ }^{13}$ B. S. Pujari, S. Gusarov, M. Brett, and A. Kovalenko, Phys. Rev. B 84, 041402(R) (2011).

${ }^{14}$ M. Klintenberg, S. Lebègue, M. I. Katsnelson, and O. Eriksson, Phys. Rev. B 81, 085433 (2010).

${ }^{15}$ M. Z. S. Flores, P. A. S. Autreto, S. B. Legoas, and D. S. Galvao, Nanotechnology 20, 465704 (2009).

${ }^{16}$ M. Neek-Amal and F. M. Peeters, Phys. Rev. B 83, 235437 (2011).

${ }^{17}$ See the recent developments in adiabatic and nonadiabatic dynamics processes in TDDFT detailed in Fundamentals of Time Dependent Density Functional Theory, edited by M. A. L. Marques, N. Maitra, F. Nogueira, E. K. U. Gross, and A. Rubio, Lecture Notes in Physics Vol. 837 (Springer, Berlin, 2011).

${ }^{18}$ O. Sugino and Y. Miyamoto, Phys. Rev. B 59, 2579 (1999); 66, 089901(E) (2002).

${ }^{19}$ The one-side H-terminated graphene on substrates (Ref. 10) cannot be a precursor of a heterogeneously terminated layer, therefore we focus on suspended graphane as a precursor for graphone.

${ }^{20}$ Y. Miyamoto and H. Zhang, Mater. Express 2, 71 (2012).

${ }^{21}$ P. Ehrenfest, Z. Phys. 45, 455 (1927).

${ }^{22}$ M. Suzuki, J. Phys. Soc. Jpn. 61, L3015 (1992).

${ }^{23}$ A. Castro, M. A. L. Marques, J. A. Alonso, G. F. Bertsch, and A. Rubio, Eur. Phys. J. D 28, 211 (2004).

${ }^{24}$ K. Taguchi, J. Haruyama, and K. Watanabe, J. Phys. Soc. Jpn. 78, 094707 (2009).

${ }^{25}$ E. Runge and E. K. U. Gross, Phys. Rev. Lett. 52, 997 (1984).

${ }^{26}$ Y. Miyamoto and H. Zhang, Phys. Rev. B 77, 165123 (2008).

${ }^{27}$ Some technical details of the calculation: Ten irreducible $k$ points were taken for the momentum-space integration and the point group of the system was $\left(C_{3 v}\right)$. A plane-wave cutoff energy of $60 \mathrm{Ry}$ is chosen to guarantee good convergence of the electronic wave functions and Hellmann-Feynman forces with the previous set of parameters. LDA is employed to deal with exchange correlation effects (Ref. 28).

${ }^{28}$ J. P. Perdew and A. Zunger, Phys. Rev. B 23, 5048 (1981).

${ }^{29}$ N. Troullier and J. L. Martins, Phys. Rev. B 43, 1993 (1991).

${ }^{30}$ Y. Miyamoto, H. Zhang, and A. Rubio, Phys. Rev. Lett. 105, 248301 (2010).

${ }^{31}$ J. Kanasaki, E. Inami, K. Tanimura, H. Ohnishi, and K. Nasu, Phys. Rev. Lett. 102, 087402 (2009).

${ }^{32}$ M. Lenner, A. Kaplan, and R. E. Palmer, Appl. Phys. Lett. 90, 153119 (2007)

${ }^{33}$ A. Roberts, D. Cormode, C. Reynolds, T. Newhouse-Illige, B. J. LeRoy, and A. S. Sandhu, Appl. Phys. Lett. 99, 051912 (2011).

${ }^{34}$ M. Currie, J. D. Caldwell, F. J. Bezares, J. Robinson, T. Anderson, H. Chun, and M. Tadjer, Appl. Phys. Lett. 99, 211909 (2011).

${ }^{35}$ The band structure is obtained with a common lattice constant within the $1 \times 1$ unit cell. The interlayer distance of the pristine and heterogeneously terminated graphene sheets is set around $5 \AA$. In this structure, a rigid shift of the Dirac cone for the graphene above or below the Fermi level is observed (see Supplemental Material at http://link.aps.org/supplemental/ 10.1103/PhysRevB.85.201409 for a supplemental figure).

${ }^{36}$ S. Latil and L. Henrard, Phys. Rev. Lett. 97, 036803 (2006).

${ }^{37}$ S. B. Kumar and J. Guo, Appl. Phys. Lett. 98, 222101 (2011).

${ }^{38} \mathrm{~A}$ fundamental question is whether any other longer asymmetric shape can produce biased desorption. This is very likely and will probably accompany heating of the carbon lattice.

${ }^{39}$ C. Spielmann, N. H. Burnett, S. Sartania, R. Koppitsch, M. Schnürer, C. Kan, M. Lenzner, P. Wobrauschek, and F. Krausz, Science 278, 661 (1997).

${ }^{40}$ M. Adachi, K. Yamane, R. Morita, and M. Yamashita, Jpn. J. Appl. Phys. 47, L1423 (2005).

${ }^{41}$ D. J. Jones, S. A. Diddams, J. K. Ranka, A. Stentz, R. S. Windeler, J. L. Hall, and S. T. Cundiff, Science 288, 635 (2000). 